\title{
CHEBYSHEV SPECTRAL COLLOCATION IN SPACE AND TIME FOR THE HEAT EQUATION*
}

\author{
S. H. LUI $^{\dagger}$ AND SARAH NATAJ ${ }^{\dagger}$
}

\begin{abstract}
Spectral methods can solve elliptic partial differential equations (PDEs) numerically with errors bounded by an exponentially decaying function of the number of modes when the solution is analytic. For timedependent problems, almost all focus has been on low-order finite difference schemes for the time derivative and spectral schemes for the spatial derivatives. This mismatch destroys the spectral convergence of the numerical solution. Spectral methods that converge spectrally in both space and time have appeared recently. This paper shows that a Chebyshev spectral collocation method of Tang and Xu for the heat equation converges exponentially when the solution is analytic. We also derive a condition number estimate of the global spectral operator. Another space-time Chebyshev collocation scheme that is easier to implement is proposed and analyzed. This paper is a continuation of the first author's earlier paper in which two Legendre space-time collocation methods were analyzed.
\end{abstract}

Key words. spectral collocation, Chebyshev collocation, space-time, time-dependent partial differential equation

AMS subject classifications. 65M70, 65L05, 35K20, 35L20, 41A10

1. Introduction. Spectral methods have been successfully used for many decades to solve elliptic PDEs. If the solution is analytic, then the numerical solution converges exponentially as a function of the number of spectral modes. For time-dependent PDEs, the most common approach is to use a low-order finite difference approximation of the time derivative and a spectral approximation of the spatial derivatives. This is not ideal since the time discretization error overwhelms the spatial discretization error. In an earlier paper [9], a Legendre collocation method in both space and time based on the work of Tang and Xu [19] was proposed for the heat equation. The method was shown to converge spectrally when the solution is analytic. A condition number estimate for the global space-time operator of $O\left(N^{4}\right)$ was derived, where $N$ is the number of spectral modes in each direction. This estimate is the same as that for the elliptic part of the operator. A second space-time method which is easier to implement and has similar performance was also proposed and analyzed. The purpose of this paper is to demonstrate spectral convergence and a $O\left(N^{4}\right)$ condition number estimate for the global space-time operator of the space-time Chebyshev collocation method. Although much of the basic framework of the theory for the methods based on the two different orthogonal polynomials are similar, the analysis for the Chebyshev case is much more difficult because of the presence of a singular weight function. In this paper, a simplified eigenvalue analysis paves the way for a condition number estimate of the Chebyshev space-time method and a similar analysis for other canonical linear PDEs ([10]). This paper and the earlier one [9] are the only ones in the literature to address the condition number of discrete global space-time operators.

Two additional contributions of this paper include sharp estimates of the eigenvalues of spectral integration and derivative matrices. It is shown that the real part of every eigenvalue of a spectral integration matrix is positive and the real part of every eigenvalue of a spectral derivative matrix is positive and bounded away from zero. See Propositions 3.4 and 4.1.

One drawback of these space-time spectral methods is that time stepping is no longer possible. The unknowns for all times must be solved simultaneously. This presents a serious problem for PDEs in three spatial dimensions and is particularly onerous for nonlinear PDEs.

* Received February 13, 2020. Accepted May 10, 2020. Published online on June 19, 2020. Recommended by Martin Gander. This work was funded by NSERC and UMGF (University of Manitoba).

$\dagger^{\dagger}$ Department of Mathematics, University of Manitoba, Winnipeg, Manitoba, Canada R3T 2N2

( $\{$ Iuish@ccumanitoba.ca, ebrahimr@myumanitoba.ca\}). 
It should be made clear that due to spectral convergence, many fewer unknowns are needed compared to finite difference/element schemes for the same error tolerance.

An early work on spectrally accurate ordinary differential equation (ODE) solvers is [4]. Among the first works on space-time spectral methods for PDEs with periodic boundary conditions are $[16,17]$. Other references include $[5,6,7,13,15,18,20,22,23]$ and the references therein. Of course, this list is incomplete. See [9] for additional papers that address space-time spectral methods and papers that attempt to couple space and time components for faster computations. We wish to add one more reference [3] that provides a good survey of algorithms that are parallel in time.

In the next section, we state the notation used in this paper and recall some basic estimates used in the analysis. Following that, we demonstrate the spectral convergence of the space-time Chebyshev collocation method of Tang and $\mathrm{Xu}$ [19] for the $1 \mathrm{D}$ heat equation in Section 3. The condition number of the method is shown to be $O\left(N^{4}\right)$. In Section 4, a similar space-time spectral collocation method which is easier to implement for more general PDEs and which exhibits nearly identical characteristics is proposed and analyzed. Some simple numerical results are illustrated in Section 5. In the final section, a conclusion and some future work are outlined. Proofs of technical results needed for the condition number estimates are collected in Appendix A.

2. Notation and basic estimates. Below, we summarize our matrix notation to be followed by the notation pertaining to spectral methods. Let $I_{n}$ denote the $n \times n$ identity matrix. For an $n \times n$ matrix $M$, let $[M]$ denote the $(n-1) \times(n-1)$ matrix obtained from $M$ by deleting the last column and row, while $\llbracket M \rrbracket$ denotes the $(n-2) \times(n-2)$ matrix obtained from $M$ by deleting the first and last columns and rows. For any complex number $a$, its complex conjugate is denoted by $\bar{a}$, and its real and imaginary parts are denoted by $\Re a$ and $\Im a$, respectively. For any matrix $M$, let $M^{T}$ and $M^{*}$ denote the transpose and complex conjugate transpose of $M$, respectively. Let $|\cdot|_{2}$ denote the vector/matrix 2-norm and $|\cdot|_{\infty}$ the vector $\infty$-norm. For positive integers $m, n$ and any $a \in \mathbb{C}^{m n}$, let $A \in \mathbb{C}^{m \times n}$ be the matrix representation of $a$, that is, the columns of $A$, stacked on top of each other, form $a$. The notation is $a=\operatorname{vec}(A)$. Finally, $\otimes$ denotes the tensor product. For matrices $X \in \mathbb{C}^{N \times N}$, $Y \in \mathbb{C}^{M \times M}$, and $z \in \mathbb{C}^{M N}$, recall that $(X \otimes Y) z=\operatorname{vec}\left(Y Z X^{T}\right)$, where $\operatorname{vec}(Z)=z$ is the vector representation of $Z$. Throughout, $C, c$ denote positive constants whose values may differ at different occurrences but are independent of $N$.

Fix a positive integer $N$. Let $P_{N}$ denote the space of polynomials of degree at most $N$ in $x$ for a fixed $t$ and degree at most $N$ in $t$ for a fixed $x$. Let $x_{0}, \ldots, x_{N}$ denote the Chebyshev Gauss-Lobatto nodes with $x_{0}=1, x_{N}=-1$, and $x_{j}$ the descending zeros of $T_{N}^{\prime}(x)$, where $1 \leq j \leq N-1$ and $T_{N}$ is the $N$ th Chebyshev polynomial. The Chebyshev Gauss-Lobatto nodes along the $t$ axis are denoted by $\left\{t_{k}\right\}$. Let

$$
x_{h}=\left[\begin{array}{c}
x_{1} \\
\vdots \\
x_{N-1}
\end{array}\right], \quad t_{h}=\left[\begin{array}{c}
t_{0} \\
\vdots \\
t_{N-1}
\end{array}\right] .
$$

Note that $x_{h}$ excludes both boundary points, while $t_{h}$ excludes only the initial point -1 . For $0 \leq j \leq N$, let $\ell_{j}$ be the Lagrange interpolant, a polynomial of degree $N$ of $x_{j}$ so that $\ell_{j}\left(x_{k}\right)=\delta_{j k}$. Recall that the Chebyshev pseudospectral derivative matrix $D \in \mathbb{R}^{(N+1) \times(N+1)}$ has entries

$$
D_{j k}=\frac{d \ell_{k}\left(x_{j}\right)}{d x}, \quad 0 \leq j, k \leq N
$$


Let $d_{h}=D(0: N-1, N)$ be the first $N$ entries of the last column of $D$. Define the Chebyshev interpolation operator as usual: for any continuous $u$,

$$
\mathcal{I}_{N} u=\sum_{j=0}^{N} u\left(x_{j}\right) \ell_{j}
$$

The following is an important property of Chebyshev quadrature: for any polynomial $v$ of degree at most $2 N-1$,

$$
\int_{-1}^{1} v(x) w(x) d x=\sum_{k=0}^{N} v\left(x_{k}\right) \rho_{k}, \quad w(x)=\frac{1}{\sqrt{1-x^{2}}},
$$

where $\left\{\rho_{k}\right\}$ is the set of weights associated with Chebyshev Gauss-Lobatto quadrature. Let $W_{h}$ be the $(N+1) \times(N+1)$ diagonal matrix whose diagonal entries are $\left\{\rho_{k}\right\}$. Denote the weighted $L^{2}$-norm of a continuous function $v$ on $\Omega:=(-1,1)^{2}$ by

$$
\|v\|:=\left(\int_{\Omega}|v(x, t)|^{2} w(x) w(t) d x d t\right)^{1 / 2} .
$$

Also, define the corresponding discrete norm

$$
\|v\|_{N}:=\left(\sum_{j, k=0}^{N} \rho_{j} \rho_{k}\left|v\left(x_{j}, t_{k}\right)\right|^{2}\right)^{1 / 2} .
$$

It is well known ([2, Inequality (5.3.2)], for instance) that the weighted $L^{2}$ - and discrete norms are equivalent for all polynomials $v$ of degree at most $N$ :

$$
\|v\| \leq\|v\|_{N} \leq 2\|v\|
$$

In case $v$ is a function of one variable, we also write

$$
\|v\|=\left(\int_{-1}^{1}|v(x)|^{2} w(x)\right)^{1 / 2} .
$$

An inverse estimate (see [2, (5.5.4)], for instance) states that there is some positive constant $c$ so that

$$
\left\|v^{\prime}\right\| \leq c N^{2}\|v\|, \quad v \in P_{N} .
$$

3. The space-time spectral collocation method of Tang-Xu. We treat the simplest case where the spatial and temporal domains are both $(-1,1)$. This is no loss of generality since this can always be accomplished by a simple change of variables. In cases where this may not be appropriate, the method can be repeatedly applied over several time intervals, for instance. Since the purpose of the paper is an analysis of the method, we shall not dwell on these and other refinements.

Consider the linear heat equation

$$
u_{t}=u_{x x}+f(x, t) \quad \text { on }(-1,1)^{2},
$$

with boundary conditions $u( \pm 1, t)=0$ and initial condition $u(x,-1)=u_{0}(x)$. We seek a numerical solution $u \in P_{N}$ at $t=1$. 
Fix an integer $N \geq 2$. We first derive the space-time Chebyshev spectral collocation method of [19]. Write

$$
\ell_{k}(t)=\sum_{q=0}^{N} \alpha_{q k} T_{q}(t), \quad 0 \leq k \leq N
$$

Let

$$
c_{k}=\left\{\begin{array}{ll}
2, & k=0, \\
1, & \text { otherwise. }
\end{array} \quad d_{k}= \begin{cases}2, & k=0, N \\
1, & \text { otherwise }\end{cases}\right.
$$

It is not difficult to show that

$$
\alpha_{q k}= \begin{cases}\frac{2}{N c_{q} d_{k}} \cos \frac{q k \pi}{N}, & 0 \leq q<N, \\ \frac{(-1)^{k}}{N d_{k}}, & q=N .\end{cases}
$$

For any real $t$, define

$$
u_{h}(t)=\left[\begin{array}{c}
u\left(x_{1}, t\right) \\
\vdots \\
u\left(x_{N-1}, t\right)
\end{array}\right], \quad f_{h}(t)=\left[\begin{array}{c}
f\left(x_{1}, t\right) \\
\vdots \\
f\left(x_{N-1}, t\right)
\end{array}\right] .
$$

A semi-discrete approximation of the heat equation is

$$
u_{h}^{\prime}(t)=\sum_{k=0}^{N}\left(A u_{h}\left(t_{k}\right)+f_{h}\left(t_{k}\right)\right) \ell_{k}(t), \quad u_{h}(-1)=u_{0 h},
$$

where $A=\llbracket D^{2} \rrbracket$ and $u_{0 h}$ are the initial data evaluated at the vector of interior Chebyshev Gauss-Lobatto points $x_{h}$, i.e., $u_{0 h}=u_{0}\left(x_{h}\right)$. Note that at the collocation point $t_{j}$, for $0 \leq j<N$,

$$
u_{h}^{\prime}\left(t_{j}\right)=A u_{h}\left(t_{j}\right)+f_{h}\left(t_{j}\right),
$$

which is precisely the system of collocation equations.

Using (3.2), it follows that

$$
u_{h}^{\prime}(t)=\sum_{q, k=0}^{N}\left(A u_{h}\left(t_{k}\right)+f_{h}\left(t_{k}\right)\right) \alpha_{q k} T_{q}(t) .
$$

Integrating in time from -1 to $t_{j}$ for some $0 \leq j<N$, we obtain

$$
\begin{aligned}
u_{h}\left(t_{j}\right)-u_{0 h}=\sum_{k=0}^{N}\left(A u_{h}\left(t_{k}\right)\right. & \left.+f_{h}\left(t_{k}\right)\right)\left[\alpha_{0 k}\left(t_{j}+1\right)+\frac{\alpha_{1 k}\left(t_{j}^{2}-1\right)}{2}\right. \\
& \left.+\sum_{q=2}^{N} \alpha_{q k}\left(\frac{T_{q+1}\left(t_{j}\right)}{2(q+1)}-\frac{T_{q-1}\left(t_{j}\right)}{2(q-1)}-\frac{(-1)^{q}}{q^{2}-1}\right)\right] .
\end{aligned}
$$


In the above formula, we used the identity

$$
T_{q}(t)=\frac{T_{q+1}^{\prime}(t)}{2(q+1)}-\frac{T_{q-1}^{\prime}(t)}{2(q-1)}, \quad q \geq 2 .
$$

The system can be represented as

$$
u_{h}\left(t_{j}\right)=A \sum_{k=0}^{N-1} B_{k j} u_{h}\left(t_{k}\right)+g_{j}, \quad 0 \leq j<N,
$$

where for $0 \leq k \leq N$,

$$
\begin{aligned}
B_{k j}= & \alpha_{0 k}\left(t_{j}+1\right)+\frac{\alpha_{1 k}\left(t_{j}^{2}-1\right)}{2} \\
& +\sum_{q=2}^{N} \alpha_{q k}\left(\frac{\cos ((q+1) \pi j / N)}{2(q+1)}-\frac{\cos ((q-1) \pi j / N)}{2(q-1)}-\frac{(-1)^{q}}{q^{2}-1}\right),
\end{aligned}
$$

and

$$
g_{j}=\sum_{k=0}^{N} B_{k j} f_{h}\left(t_{k}\right)+B_{N j} A u_{0 h}+u_{0 h} .
$$

We record the following identity for future reference:

$$
B_{k j}=\int_{-1}^{t_{j}} \ell_{k}(t) d t=\sum_{q=0}^{N} \alpha_{q k} \int_{-1}^{t_{j}} T_{q}(t) d t .
$$

Let $V_{h}, G_{h} \in \mathbb{R}^{(N-1) \times N}$ be the matrices whose $j$ th column is $u_{h}\left(t_{j}\right)$ and $g_{j}, 0 \leq j<N$, respectively. Then the system becomes

$$
V_{h}=A V_{h} B+G_{h},
$$

where $B \in \mathbb{R}^{N \times N}$ with entries $B_{k j}, 0 \leq k, j \leq N-1$. Let $v_{h}=\operatorname{vec}\left(V_{h}\right), g_{h}=\operatorname{vec}\left(G_{h}\right)$, and

$$
\mathcal{A}_{h}=\left(I_{N} \otimes I_{N-1}\right)-\left(B^{T} \otimes A\right) .
$$

Then (3.5) is equivalent to $\mathcal{A}_{h} v_{h}=g_{h}$.

We begin with some preliminary results. The first two state that $B^{T}$ is a discrete integration operator in two different senses: it exactly integrates polynomials of degree at most $N$ evaluated at the collocation points and its inverse differs from $[D]$ by a rank-one matrix. These facts are hardly surprising by the way $B$ was derived in (3.4).

LEMMA 3.1. Let $N \geq 1$ and $v$ be a complex polynomial of degree at most $N$ so that $v(-1)=0$. Then

$$
B^{T} v\left(t_{h}\right)=\int_{-1}^{t_{h}} v(t) d t
$$

Proof. The proof is exactly the same as that for the Legendre case and is omitted. See [9].

Lemma 3.2. Let $N \geq 1$. Then $B^{T}-[D]^{-1}$ is a rank-one matrix. 
Proof. Let $\left\{a_{k}\right\}$ be arbitrary complex constants so that

$$
u(t)=\sum_{k=0}^{N} a_{k} t^{k}, \quad u(-1)=0 .
$$

Define $u_{h}=u\left(t_{h}\right)$. Let 1 be the vector of all ones. By the above lemma,

$$
\begin{aligned}
{[D] B^{T} u_{h} } & =[D] \int_{-1}^{t_{h}} \sum_{k=0}^{N-1} a_{k} t^{k} d t+[D] \int_{-1}^{t_{h}} a_{N} t^{N} d t \\
& =\sum_{k=0}^{N-1} a_{k} t_{h}^{k}+\frac{a_{N}}{N+1}[D]\left(t_{h}^{N+1}-(-1)^{N+1} \mathbf{1}\right) \\
& =u_{h}+a_{N}\left(\frac{[D]}{N+1}\left(t_{h}^{N+1}+(-1)^{N} \mathbf{1}\right)-t_{h}^{N}\right) .
\end{aligned}
$$

Thus $[D] B^{T}-I_{N}$ is a rank-one matrix which depends on $a_{N}$ but is independent of all other $a_{j}, 0 \leq j<N$.

The third lemma states that when applied to an analytic function evaluated at the collocation points, the quadrature error is exponentially small.

LEMMA 3.3. Let $N \geq 1$ and $z$ be analytic with $z(-1)=0$. Then

$$
\left|B^{T} z\left(t_{h}\right)-\int_{-1}^{t_{h}} z(t) d t\right|_{2} \leq c N^{1 / 2} e^{-C N}
$$

where c depends on $z$ but is independent of $N$.

Proof. Let $L$ denote the quantity at the left-hand side of the inequality of the lemma. Then

$$
\begin{aligned}
L & =\left|\left[B^{T}\left(\mathcal{I}_{N} z\right)\left(t_{h}\right)-\int_{-1}^{t_{h}}\left(\mathcal{I}_{N} z\right)(t) d t\right]+\int_{-1}^{t_{h}}\left(\mathcal{I}_{N} z-z\right)(t) d t\right|_{2} \\
& \leq 0+\sqrt{2 N}\left\|\mathcal{I}_{N} z-z\right\|_{L^{2}(-1,1)} \\
& \leq c N^{1 / 2} e^{-C N} .
\end{aligned}
$$

Note that the term inside the square brackets is zero due to Lemma 3.1, while the last inequality is a Chebyshev interpolation error estimate for analytic functions. See [8, (5.45)], for instance.

The following results are needed to estimate the condition number of the method. The proof of the first one, one of the main technical results of this paper, is postponed to the appendix.

Proposition 3.4. Let $N \geq 1$. The real part of every eigenvalue of $B^{T}$ is positive.

LEMMA 3.5. Let $N \geq 1$. Then $\left|B^{T}\right|_{2} \leq c$, with $c$ a positive constant independent of $N$.

Proof. Let $z_{h}$ be a unit $N$-vector so that $\left|B^{T} z_{h}\right|_{2}=\left|B^{T}\right|_{2}$. Let $z$ be a polynomial of degree at most $N$ with $z(-1)=0$ and $z_{h}=z\left(t_{h}\right)$. By Lemma 3.1,

$$
\begin{aligned}
\left|B^{T} z_{h}\right|_{2}^{2} & =\left|\int_{-1}^{t_{h}} z(t) d t\right|_{2}^{2}=\sum_{k=0}^{N-1}\left(\int_{-1}^{t_{k}} z(t) w^{1 / 2}(t) \cdot w^{-1 / 2}(t) d t\right)^{2} \\
& \leq \sum_{k=0}^{N-1} \int_{-1}^{t_{k}} z^{2}(t) w(t) d t \int_{-1}^{1} \sqrt{1-t^{2}} d t \leq \frac{\pi N}{2} \int_{-1}^{1} z^{2}(t) w(t) d t \\
& \leq \frac{\pi N}{2}\left|\left[W_{h}^{1 / 2}\right] z_{h}\right|_{2}^{2} \leq c
\end{aligned}
$$


The penultimate inequality is due to the equivalence of the discrete and weighted $L^{2}$-norms (2.2), while the last inequality follows from the fact that the weights satisfy $\rho_{k} \leq c N^{-1}$ for all $k$.

The next lemma is well known; see [2, Inequality (7.3.5)] or [21], for instance.

Lemma 3.6. Let $N \geq 2$. Then the eigenvalues of $-\llbracket D^{2} \rrbracket$ are real, bounded below by $c$ and above by $C N^{4}$, where $c$ and $C$ are positive and independent of $N$.

LEMMA 3.7. Let $N \geq 2$ and $u$ be a function analytic in some open set in the complex plane containing the real interval $[-1,1]$ and $u( \pm 1)=0$. Let $A=\llbracket D^{2} \rrbracket$, where $D$ is the Chebyshev pseudospectral derivative matrix. Then

$$
\left|\left(A u\left(x_{h}\right)-u^{\prime \prime}\left(x_{h}\right)\right)\right|_{\infty} \leq c N^{3} e^{-C N} .
$$

Proof. Recall the definition of the interpolation operator in (2.1). Observe that the identity $\left(\mathcal{I}_{N} u\right)^{\prime \prime}\left(x_{h}\right)=A u\left(x_{h}\right)$ holds. The result follows from the estimate ([12])

$$
\left|\left(\mathcal{I}_{N} u-u\right)^{\prime \prime}\left(x_{h}\right)\right|_{\infty} \leq c N^{3} e^{-C N} .
$$

For any $t$, define the error vector

$$
e_{h}(t)=u_{h}(t)-u\left(x_{h}, t\right),
$$

where $u$ is the solution of the heat equation (3.1) and $u_{h}$ is the solution of (3.3). For $0 \leq k<N$,

$$
\begin{aligned}
e_{h}^{\prime}\left(t_{k}\right) & =u_{h}^{\prime}\left(t_{k}\right)-u_{t}\left(x_{h}, t_{k}\right) \\
& =A u_{h}\left(t_{k}\right)+f\left(x_{h}, t_{k}\right)-\left(u_{x x}\left(x_{h}, t_{k}\right)+f\left(x_{h}, t_{k}\right)\right) \\
& =A u_{h}\left(t_{k}\right)-A u\left(x_{h}, t_{k}\right)+A u\left(x_{h}, t_{k}\right)-u_{x x}\left(x_{h}, t_{k}\right) \\
& =A e_{h}\left(t_{k}\right)+r\left(t_{k}\right),
\end{aligned}
$$

where $r\left(t_{k}\right)=A u\left(x_{h}, t_{k}\right)-u_{x x}\left(x_{h}, t_{k}\right)$.

Let $E_{h}:=E_{h}\left(t_{h}\right)$ be the long vector consisting of the vectors $e_{h}\left(t_{k}\right)$, for $k=0$ to $N-1$, stacked one on top of the other. Similarly, define $\tilde{R}_{h}$ as the long vector consisting of the vectors $r\left(t_{k}\right)$ :

$$
E_{h}=\left[\begin{array}{c}
e_{h}\left(t_{0}\right) \\
\vdots \\
e_{h}\left(t_{N-1}\right)
\end{array}\right], \quad \tilde{R}_{h}=\left[\begin{array}{c}
r\left(t_{0}\right) \\
\vdots \\
r\left(t_{N-1}\right)
\end{array}\right]
$$

The system $e_{h}^{\prime}\left(t_{k}\right)=A e_{h}\left(t_{k}\right)+r\left(t_{k}\right)$ for all $k$ can be more compactly represented as

$$
E_{h}^{\prime}\left(t_{h}\right)=\left(I_{N} \otimes A\right) E_{h}+\tilde{R}_{h} .
$$

Applying $B^{T} \otimes I_{N-1}$ on both sides leads to

$$
E_{h}=\left(B^{T} \otimes A\right) E_{h}+\left(B^{T} \otimes I_{N-1}\right) \tilde{R}_{h}+\delta,
$$

where $\delta$ is a long vector with matrix representation $\Phi$. Let $\Phi_{j}$ be the $j$ th row of $\Phi$. According to Lemma 3.3, $\left|\Phi_{j}\right|_{2} \leq c N^{1 / 2} e^{-C N}$. The above equality can also be written as

$$
\mathcal{A}_{h} E_{h}=R_{h}, \quad R_{h}=\left(B^{T} \otimes I_{N-1}\right) \tilde{R}_{h}+\delta .
$$

See (3.5). 
THEOREM 3.8. Let $N \geq 2$ and $\lambda$ be an eigenvalue of $\mathcal{A}_{h}$. Then

$$
1 \leq|\lambda| \leq c N^{4}
$$

Proof. Let $\left(v_{h}, \lambda\right)$ be an eigenpair of the matrix $\mathcal{A}_{h}$. Then it follows from (3.7) that $(\lambda-1) v_{h}=-\left(B^{T} \otimes A\right) v_{h}$, or

$$
\lambda-1=\gamma_{j} \mu_{k},
$$

where $\gamma_{j}$ is some eigenvalue of $B^{T}$ and $\mu_{k}$ is some eigenvalue of $-A$. The lower bound $|\lambda|>1$ follows from Proposition 3.4 and Lemma 3.6. From (3.8), an upper bound of $|\lambda|$ follows from Lemmas 3.5 and 3.6:

$$
|\lambda| \leq 1+\left|\gamma_{j}\right| \mu_{k} \leq 1+c N^{4}
$$

We are able to derive the same upper bound for the magnitude of the eigenvalue using the technique of [9] but not the lower bound. The technique employed here is much simpler conceptually because the analysis reduces to an eigenvalue analysis of $B^{T}$ and $A$.

THEOREM 3.9. For any integer $N \geq 2$, let $u$ be the solution of the heat equation (3.1). Assume that $u(x, t)$ is separately analytic in each variable. Let $u_{h}$ be the solution of (3.4) and $E_{h}$ be the long error vector defined in (3.6). Then

$$
\left|W^{1 / 2} E_{h}\right|_{2} \leq c N^{3.5} e^{-C N} .
$$

Proof. The proof uses Lemmas 3.7 and 3.5. It is similar to the proof given for the Legendre case in [9] and is not presented here. See [11].

We remark that for $f \in P_{N}$ and $f_{h}$, the long vector of $f$ evaluated at the collocation points satisfies

$$
\begin{aligned}
\left(\int_{-1}^{1} \int_{-1}^{1}|f(x, t)|^{2} w(x) d x w(t) d t\right)^{1 / 2} & \leq\left|W^{1 / 2} f_{h}\right|_{2} \\
& \leq 2\left(\int_{-1}^{1} \int_{-1}^{1}|f(x, t)|^{2} w(x) d x w(t) d t\right)^{1 / 2}
\end{aligned}
$$

using the equivalence of the weighted $L^{2}$ - and the discrete norms. This is the main reason for measuring the error in the discrete norm.

To measure the difficulty to solve a linear system with the coefficient matrix $M$, we sometimes use the spectral condition number, defined by

$$
\kappa(M)=\frac{\max _{\lambda \in \Lambda(M)}|\lambda|}{\min _{\lambda \in \Lambda(M)}|\lambda|}
$$

where $\Lambda(M)$ is the spectrum of $M$. Using the result of Theorem 3.8, it is easy to estimate the spectral condition number of the space-time spectral collocation method:

COROllary 3.10. Let $N \geq 2$. Then

$$
\kappa\left(\mathcal{A}_{h}\right) \leq c N^{4} .
$$

Note that a direct solver for (3.5) is the method of Bartels and Stewart [1]. 


\section{ETNA}

Kent State University and

Johann Radon Institute (RICAM)

4. A second space-time collocation method. We now give an alternate space-time spectral numerical method for the solution $u \in P_{N}$ of the heat equation (3.1). The spectral equations are

$$
\left(I_{N+1} \otimes D\right) u_{h}=\left(D^{2} \otimes I_{N+1}\right) u_{h}+f_{h},
$$

where $u_{h}$ and $f_{h}$ are the vectors of $u$ and $f$, respectively, evaluated at the collocation points. (The order of the variables is different from that of the first method for historical reasons.) Let $\hat{u}_{h}$ denote the vector $u_{h}$ obtained by deleting the components corresponding to boundary points and initial points, and similarly, $\hat{f}_{h}$ is the vector $f_{h}$ after removing the components corresponding to boundary points and initial points. The linear equation to be solved becomes

$$
A_{h} \hat{u}_{h}=\hat{f}_{h}-\left(u_{0 h} \otimes d_{h}\right), \quad A_{h}=\left(I_{N-1} \otimes[D]\right)-\left(\llbracket D^{2} \rrbracket \otimes I_{N}\right) .
$$

Let $\operatorname{vec}\left(U_{h}\right)=\hat{u}_{h}$ and $\operatorname{vec}\left(F_{h}\right)=\hat{f}_{h}-\left(u_{0 h} \otimes d_{h}\right)$. Here $U_{h}$ and $F_{h}$ are $N \times(N-1)$ matrices. Then the above equation is equivalent to the Sylvester equation $[D] U_{h}-U_{h} \llbracket D^{2} \rrbracket^{T}=F_{h}$. This matrix system can be solved in $O\left(N^{3}\right)$ operations by the algorithm of Bartels and Stewart.

Let us see if there is any relation between this formulation and (3.5). Recall that $A=\llbracket D^{2} \rrbracket$. If the two methods are equivalent, that is, they yield the same matrix equation and, of course, have the same solution (under exact arithmetic), then $V_{h}=U_{h}^{T}$. Taking the transpose of the second system results in $V_{h}[D]^{T}-A V_{h}=F_{h}^{T}$ or $V_{h}-A V_{h}[D]^{-T}=F_{h}^{T}[D]^{-T}$. Unfortunately, from Lemma 3.2, $[D]^{-T}$ is not identical to $B$ (this can also be verified by an explicit computation for small values of $N$ ), and so the two methods are different.

In [9], it was mentioned that the second method for Legendre space-time collocation is easier to implement for more complicated PDEs. The same reasoning also applies here to the Chebyshev case.

Next we state two useful results, where the first one is a sharpening of a result proved in [14] in the context of stability theory of a linear hyperbolic PDE. The sharper result is not needed in this paper but is crucial in the upcoming work [10]. Its proof is postponed to the appendix.

PROPOSITION 4.1. Let $N \geq 1$. The real part of every eigenvalue of $[D]$ is positive and bounded away from zero.

Lemma 4.2. Let $N \geq 1$ and $\lambda$ be an eigenvalue of $[D]$. Then $|\lambda| \leq c N^{2}$.

Proof. An upper bound for the magnitude of an eigenvalue of $D$ is well known and also equals $c N^{2}$. Its proof is very similar to the proof of this lemma which is included here for completeness.

Let $v_{h}$ be an eigenvector corresponding to $\lambda$ and $v$ be the unique polynomial of degree at most $N$ so that $v(-1)=0$ and $v\left(t_{h}\right)=v_{h}$. Note that $[D] v_{h}=\lambda v_{h}$ and $[D] v_{h}=v^{\prime}\left(t_{h}\right)$, with the latter holding true due to the fact that $v$ is a polynomial of degree at most $N$ and the action of $[D]$ on $v\left(t_{h}\right)$ gives its derivative exactly at the collocation points. It follows that $v^{\prime}\left(t_{j}\right)=\lambda v\left(t_{j}\right)$, for $0 \leq j<N$, and so

$$
\sum_{j=0}^{N} v^{\prime}\left(t_{j}\right) \overline{v\left(t_{j}\right)} \rho_{j}=\lambda \sum_{j=0}^{N}\left|v\left(t_{j}\right)\right|^{2} \rho_{j} .
$$

Note that the above two terms corresponding to $j=N$ are both zero since $v(-1)=0$. Since Chebyshev quadrature is exact for polynomials of degree at most $2 N-1$, the left-hand side is equal to the integral

$$
\int_{-1}^{1} v^{\prime}(t) \overline{v(t)} w(t) d t \leq \sqrt{\int_{-1}^{1}\left|v^{\prime}\right|^{2} w} \sqrt{\int_{-1}^{1}|\bar{v}|^{2} w} \leq c N^{2} \int_{-1}^{1}|v|^{2} w,
$$


with the last inequality holding true due to the inverse estimate (2.3). Thus,

$$
|\lambda|=\frac{\left|\int_{-1}^{1} v^{\prime} \bar{v} w\right|}{\sum_{j=0}^{N}\left|v\left(t_{j}\right)\right|^{2} \rho_{j}} \leq c N^{2} \frac{\int_{-1}^{1}|v|^{2} w}{\sum_{j=0}^{N}\left|v\left(t_{j}\right)\right|^{2} \rho_{j}} \leq C N^{2}
$$

by the equivalence of the discrete and the weighted $L^{2}$-norms.

The theorem below states that the spectral condition number of the discrete spectral differentiation operator scales like $O\left(N^{4}\right)$.

THEOREM 4.3. Let $N \geq 2$. Let $A_{h}$ be the Chebyshev spectral collocation matrix defined above. Then

$$
\kappa\left(A_{h}\right) \leq C N^{4}
$$

Proof. Let $\left\{\gamma_{j}\right\}$ be the set of eigenvalues of $[D]$ and $\left\{\mu_{j}\right\}$ be those of $-\llbracket D^{2} \rrbracket$. From (4.1), it follows that for some $j, k$,

$$
\lambda=\gamma_{j}+\mu_{k} .
$$

From Proposition 4.1 and Lemma 3.6, $\Re \gamma_{j}, \mu_{k} \geq c$, for some positive constant $c$ independent of $N$. Hence $\Re \lambda \geq 2 c$, which implies that $|\lambda| \geq 2 c$. From Lemmas 4.2 and 3.6, it follows that

$$
|\lambda| \leq C N^{2}+c N^{4} \leq C_{1} N^{4} .
$$

Combine the above two inequalities to obtain

$$
\kappa\left(A_{h}\right) \leq c N^{4} .
$$

The convergence analysis is very similar to the one in the previous section. Let $v$ be analytic in a region in the complex plane containing the real interval $[-1,1]$ and $v(-1)=0$. Let $0 \leq k<N$ and $\epsilon_{k}=\left(v-\mathcal{I}_{N} v\right)^{\prime}\left(t_{k}\right)$. From [12], it is known that $\left|\epsilon_{k}\right|_{\infty} \leq c N^{2} e^{-C N}$. Observe that

$$
\begin{aligned}
v^{\prime}\left(t_{k}\right) & =\left(\mathcal{I}_{N} v\right)^{\prime}\left(t_{k}\right)+\left(v-\mathcal{I}_{N} v\right)^{\prime}\left(t_{k}\right) \\
& =\left([D]\left(\mathcal{I}_{N}\right) v\left(t_{h}\right)\right)_{k}+\epsilon_{k}=\left([D] v\left(t_{h}\right)\right)_{k}+\epsilon_{k} .
\end{aligned}
$$

Recall the error equation

$$
e^{\prime}\left(t_{k}\right)=A e\left(t_{k}\right)+r\left(t_{k}\right), \quad 0 \leq k<N,
$$

where $r\left(t_{k}\right)=A u\left(x_{h}, t_{k}\right)-u_{x x}\left(x_{h}, t_{k}\right)$. Let $1 \leq j \leq N-1$ and $e_{j}\left(t_{k}\right)$ refer to the $j$ th component of $e\left(t_{k}\right)$, and define

$$
e_{j}\left(t_{h}\right)=\left[\begin{array}{c}
e_{j}\left(t_{0}\right) \\
\vdots \\
e_{j}\left(t_{N-1}\right)
\end{array}\right], \quad E_{h}=\left[\begin{array}{c}
e_{1}\left(t_{h}\right) \\
\vdots \\
e_{N-1}\left(t_{h}\right)
\end{array}\right], \quad \tilde{R}_{h}=\left[\begin{array}{c}
r_{1}\left(t_{h}\right) \\
\vdots \\
r_{N-1}\left(t_{h}\right)
\end{array}\right] .
$$

Note that these vectors are the same as those defined in (3.6) except for a different ordering. Then, from the previous calculation, we have

$$
\left([D] e_{j}\left(t_{h}\right)\right)_{k}+\epsilon_{j k}=e_{j}^{\prime}\left(t_{k}\right)=\left(A e_{h}\left(t_{k}\right)\right)_{j}+r_{j}\left(t_{k}\right),
$$




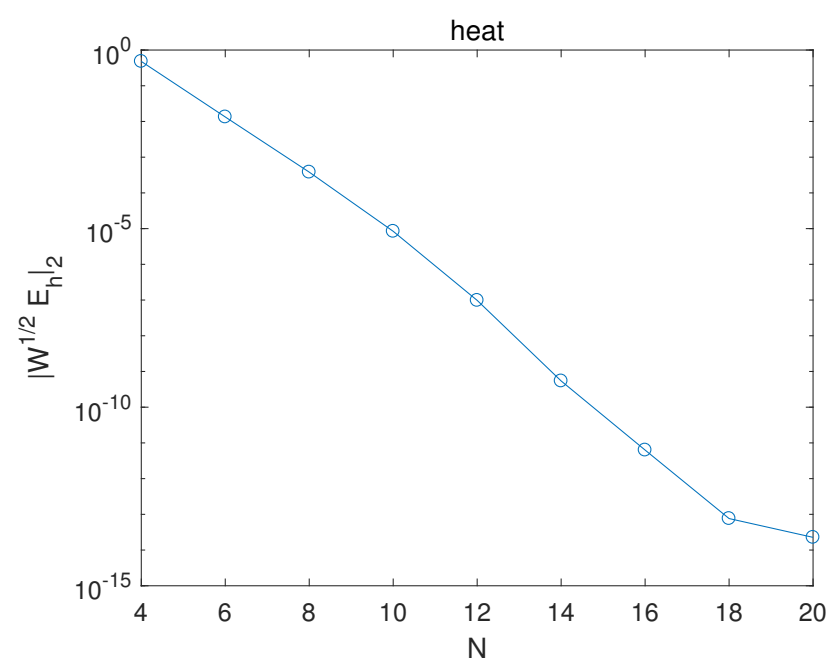

FIG. 5.1. Convergence of the Chebyshev collocation method $\left(\mathcal{A}_{h}\right)$ for the heat equation.

where $\left|\epsilon_{j k}\right|_{\infty} \leq N^{2} e^{-C N}$, or

$$
A_{h} E_{h}=R_{h}:=\tilde{R}_{h}-\epsilon,
$$

where $\epsilon$ is a long vector formed by stacking together the vectors $\epsilon_{j}=\left[\epsilon_{j 0}, \ldots, \epsilon_{j, N-1}\right]^{T}$. Using exactly the same analysis as before, the following convergence result can be shown:

THEOREM 4.4. For any integer $N \geq 2$, let $u$ be the solution of the heat equation (3.1). Assume that $u(x, t)$ is separately analytic in each variable. Then

$$
\left|W^{1 / 2} E_{h}\right|_{2} \leq c N^{3.5} e^{-C N} .
$$

5. Numerical results. We have implemented a very simple Chebyshev collocation MATLAB program. First consider the heat equation

$$
u_{t}=u_{x x}+f
$$

with boundary conditions $u( \pm 1, t)=0$ and initial condition $u(x,-1)=u_{0}(x)$. Take $f$ so that the exact solution is $u(x, t)=e^{x+t} \sin (\pi t / 2) \sin \pi x$. For the method of Tang and $\mathrm{Xu}$, the spectral convergence is clearly illustrated in Figure 5.1. Note that the error $E_{h}$ is $O\left(10^{-14}\right)$ at $N=18$, which corresponds to a system with 306 unknowns. The spectrum of the discrete heat operator $\mathcal{A}_{h}$ for the case $N=60$ and a plot of the spectral condition numbers as a function of $N$ are displayed in Figure 5.2. The corresponding figures for the second method $\left(A_{h}\right)$ are given in Figures 5.3 and 5.4.

We also performed some numerical experiments for some nonlinear PDEs: the AllenCahn equation and the viscous Burgers' equations. Since the numerical results are very similar to the Legendre case, we do not include those in this paper. See [11].

It is straightforward to extend the methods to two spatial dimensions. As an illustration, take $f$ so that the solution of the $2 \mathrm{D}$ heat equation $u_{t}=\Delta u+f$ on the spatial domain $(-1,1)^{2}$ is

$$
u(x, y, t)=e^{x+y+t+1} \sin \pi x \sin \pi y .
$$

The convergence for the second method of Section 4 is illustrated in Figure 5.5. 

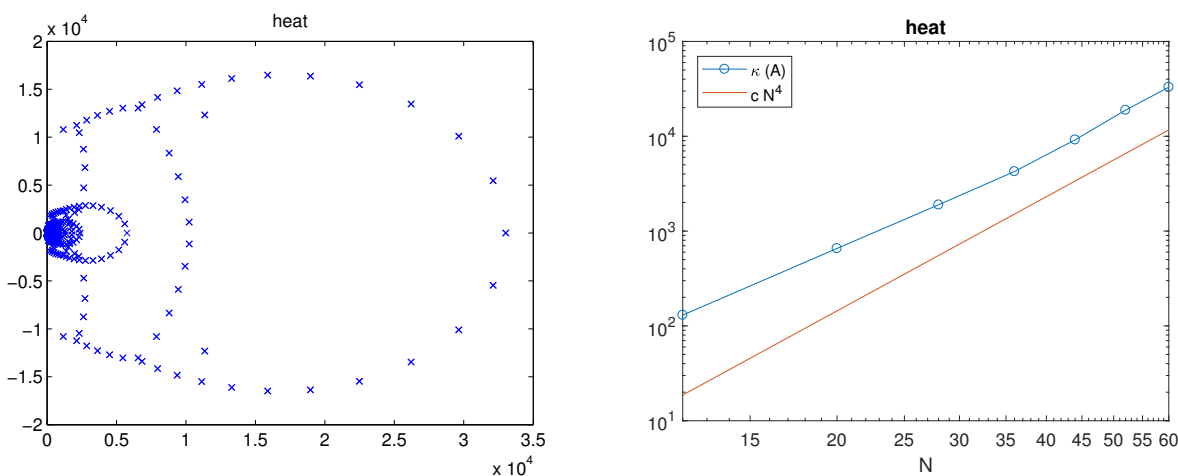

FIG. 5.2. Spectrum (left) and spectral condition number (right) for the heat operator $\mathcal{A}_{h}$.

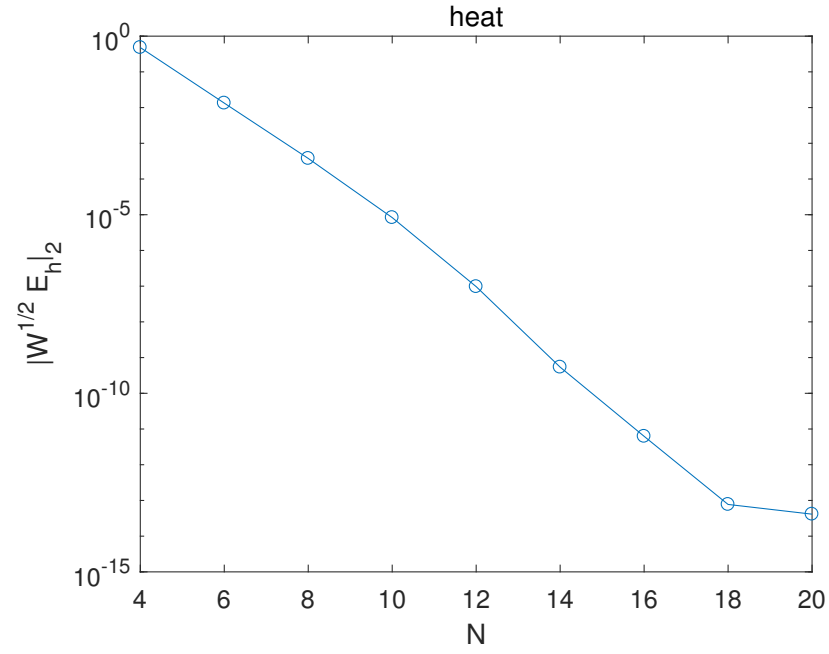

FIG. 5.3. Convergence of the Chebyshev collocation method $\left(A_{h}\right)$ for the heat equation.
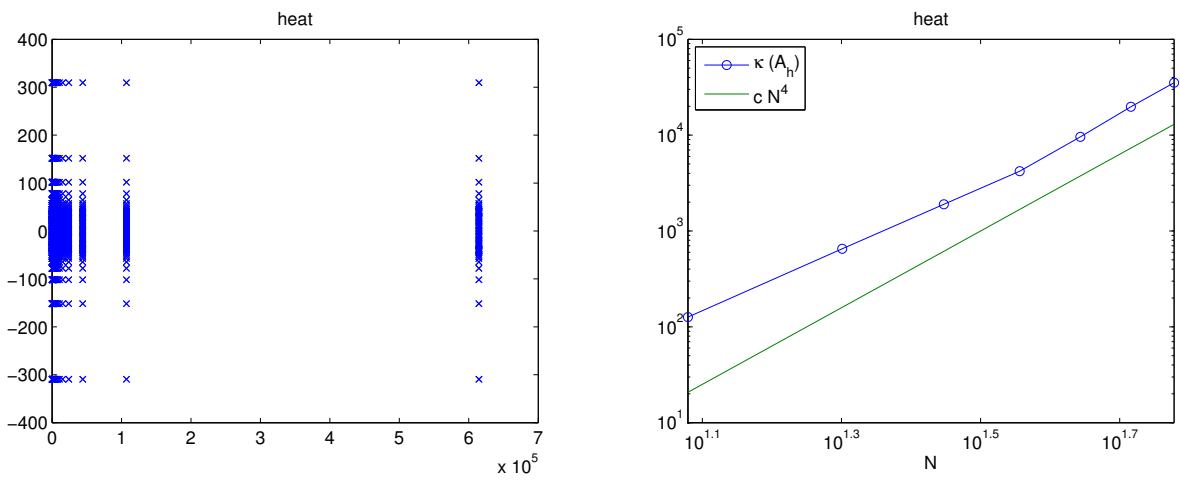

FIG. 5.4. Spectrum (left) spectral condition number (right) for the heat operator $A_{h}$. 


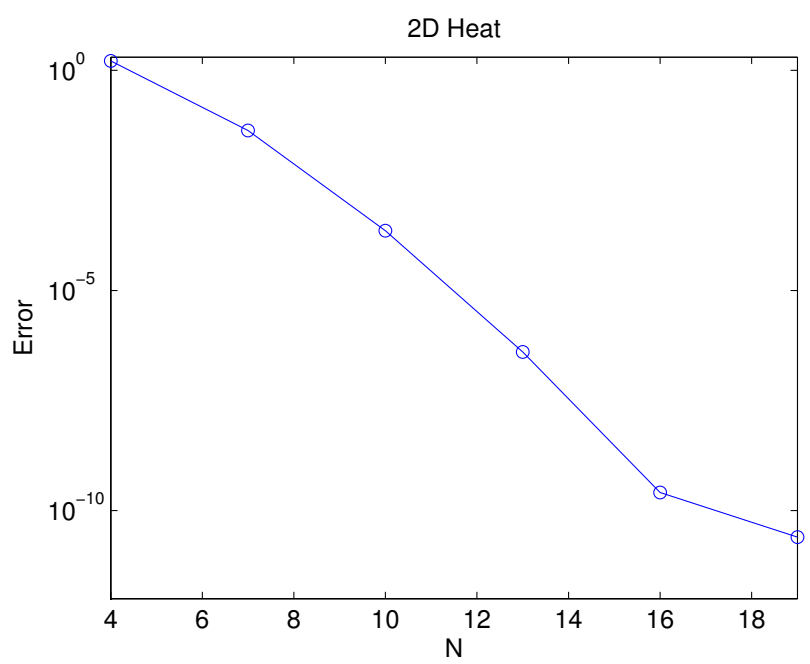

FIG. 5.5. Convergence of $2 D$ heat equation. The error is the maximum error at the final time $t=1$.

6. Discussion and conclusion. In this paper, we have shown that the space-time Chebyshev collocation method of Tang and Xu [19] converges spectrally in both space and time for the heat equation. The condition number of this method is shown to be bounded by $O\left(N^{4}\right)$. We have also proposed another space-time spectral collocation which is easier to implement and has similar characteristics as the first one. The analysis relies on new sharp estimates of the spectra of $[D]$ and $B^{T}$. Some simple numerical experiments verify the theoretical results: the space-time spectral convergence and an $O\left(N^{4}\right)$ condition number of both methods. Numerical results for the viscous Burgers' and the Allen-Cahn equations demonstrate the potential of this method for nonlinear PDEs.

In [10], we have extended our analysis to other standard linear PDEs (Schrödinger, Airy, wave, and beam equations) and conducted numerical experiments for common nonlinear PDEs (nonlinear reaction diffusion equation from combustion, nonlinear Schrödinger, KdV, Sine-Gordon, Kuramoto-Shivashinsky and Cahn-Hilliard) with similar results. It is remarkable that space-time spectral methods work so well for these different classical PDEs with different features: diffusion, dispersion, nonlinear advection, etc.

Space-time spectral methods are extremely robust methods, which converge spectrally for most standard linear PDEs with standard boundary conditions. They deserve more investigations, especially more sophisticated algorithms to speed up the linear algebra.

Appendix A. Proofs of the propositions. In this appendix, we prove Propositions 3.4 and 4.1.

First, the following preliminary result due to [14] is useful. Since no proof was given there, we include it here for completeness.

LEMMA A.1. Let $N \geq 1$. If $f=\sum_{k=0}^{4 N-1} b_{k} T_{k}$ for some complex constants $b_{k}$, then

$$
\sum_{j=0}^{N} \rho_{j} f\left(t_{j}\right)=\int_{-1}^{1} f(t) w(t) d t+\pi b_{2 N}
$$


Proof. Using the definition of $f$,

$$
\begin{aligned}
\sum_{j=0}^{N} \rho_{j} f\left(t_{j}\right)- & \int_{-1}^{1} f(t) w(t) d t=\sum_{j=0}^{N} \rho_{j} \sum_{k=0}^{4 N-1} b_{k} T_{k}\left(t_{j}\right)-\int_{-1}^{1} \sum_{k=0}^{4 N-1} b_{k} T_{k}(t) w(t) d t \\
=\sum_{k=0}^{2 N-1} b_{k}\left[\sum_{j=0}^{N} \rho_{j} T_{k}\left(t_{j}\right)-b_{k} \int_{-1}^{1} T_{k}(t) w(t) d t\right] & \\
+ & b_{2 N}\left[\sum_{j=0}^{N} \rho_{j} T_{2 N}\left(t_{j}\right)-\int_{-1}^{1} T_{2 N}(t) w(t) d t\right] \\
& +\sum_{k=2 N+1, k \text { even }}^{4 N-1} b_{k}\left[\sum_{j=0}^{N} \rho_{j} T_{k}\left(t_{j}\right)-\int_{-1}^{1} T_{k}(t) w(t) d t\right] \\
& +\sum_{k=2 N+1, k \text { odd }}^{4 N-1} b_{k}\left[\sum_{j=0}^{N} \rho_{j} T_{k}\left(t_{j}\right)-\int_{-1}^{1} T_{k}(t) w(t) d t\right] \\
=: S_{1}+ & S_{2}+S_{3}+S_{4} .
\end{aligned}
$$

$S_{1}=0$ since Chebyshev-Gaussian quadrature is exact for any polynomial of degree at most $2 N-1$. Using the identity

$$
2 T_{m} T_{n}=T_{m+n}+T_{|m-n|},
$$

$T_{2 N}=2 T_{N}^{2}-1$ follows immediately. Then, for the term $S_{2}$, we find

$$
\begin{gathered}
\sum_{j=0}^{N} \rho_{j} T_{2 N}\left(t_{j}\right)-\int_{-1}^{1} T_{2 N}(t) w(t) d t=\sum_{j=0}^{N} \rho_{j}\left(2 T_{N}^{2}\left(t_{j}\right)-1\right)-\int_{-1}^{1}\left(2 T_{N}^{2}(t)-1\right) w(t) d t \\
=2\left(\sum_{j=0}^{N} \rho_{j} T_{N}^{2}\left(t_{j}\right)-\int_{-1}^{1} T_{N}^{2}(t) w(t) d t\right)-\left(\sum_{j=0}^{N} \rho_{j}-\int_{-1}^{1} w(t) d t\right) \\
=2\left(\pi-\frac{\pi}{2}\right)-0=\pi .
\end{gathered}
$$

In the above formulas, the definition of the Chebyshev Gauss-Lobatto points $t_{j}=\cos (j \pi / N)$ has been used to evaluate the penultimate sum:

$$
\begin{aligned}
\sum_{j=0}^{N} \rho_{j} T_{N}^{2}\left(t_{j}\right) & =\sum_{j=0}^{N} \rho_{j} \cos ^{2}\left(N \cos ^{-1}\left[\cos \left(\frac{\pi j}{N}\right)\right]\right) \\
& =\sum_{j=0}^{N} \rho_{j} \cos ^{2}(\pi j)=\sum_{j=0}^{N} \rho_{j}=\int_{-1}^{1} w(t) d t=\pi .
\end{aligned}
$$

Therefore $S_{2}=\pi b_{2 N}$.

For $S_{3}$, assume $k=2 N+2 p$, for $1 \leq p \leq N-1$. Then,

$$
\begin{aligned}
\sum_{j=0}^{N} \rho_{j} T_{N+p}^{2}\left(t_{j}\right) & =\sum_{j=0}^{N} \rho_{j} \cos ^{2}\left((N+p) \frac{\pi j}{N}\right) \\
& =\sum_{j=0}^{N} \rho_{j} \cos ^{2}\left(\pi j+p \frac{\pi j}{N}\right)=\int_{-1}^{1} T_{p}^{2}(t) w(t) d t .
\end{aligned}
$$


From (A.1), $T_{2 N+2 p}=2 T_{N+p}^{2}-1$, and so

$$
\begin{aligned}
& \sum_{j=0}^{N} \rho_{j} T_{2 N+2 p}\left(t_{j}\right)-\int_{-1}^{1} T_{2 N+2 p}(t) w(t) d t \\
& \quad=\sum_{j=0}^{N} \rho_{j}\left(2 T_{N+p}^{2}\left(t_{j}\right)-1\right)-\int_{-1}^{1}\left(2 T_{N+p}^{2}(t)-1\right) w(t) d t \\
& \quad=2\left(\sum_{j=0}^{N} \rho_{j} T_{p}^{2}\left(t_{j}\right)-\int_{-1}^{1} T_{p}^{2}(t) w(t) d t\right)-\left(\sum_{j=0}^{N} \rho_{j}-\int_{-1}^{1} w(t) d t\right) \\
& \quad=0 .
\end{aligned}
$$

Consequently, $S_{3}=0$.

Finally, assume $k=2 N+2 p+1$, for $0 \leq p \leq N-1$. Then,

$$
\begin{aligned}
\sum_{j=0}^{N} \rho_{j} T_{2 N+2 p+1}\left(t_{j}\right)=\sum_{j=0}^{N} \rho_{j} \cos \left((2 N+2 p+1) \frac{\pi j}{N}\right) \\
=\sum_{j=0}^{N} \rho_{j} \cos \left(\frac{(2 p+1) \pi j}{N}\right)=\int_{-1}^{1} T_{2 p+1}(t) w(t) d t=0,
\end{aligned}
$$

since $T_{2 p+1}$ is an odd function. By the same reason,

$$
\int_{-1}^{1} T_{2 N+2 p+1}(t) w(t)=0
$$

Therefore

$$
\sum_{j=0}^{N} \rho_{j} T_{2 N+2 p+1}\left(t_{j}\right)-\int_{-1}^{1} T_{2 N+2 p+1}(t) w(t) d t=0,
$$

implying that $S_{4}=0$. This completes the proof.

Next we give a proof of Proposition 4.1. As mentioned before, it is an improvement of a result due to [14] which showed that the real part of every eigenvalue of $[D]$ is positive. The technique of proof is directly relevant for the proof of Proposition 3.4.

Proof of Proposition 4.1. Let $\lambda$ be an eigenvalue of $[D]$ and $v$ be a polynomial of degree $N$ so that $v(-1)=0$ :

$$
v=\sum_{k=0}^{N} a_{k} T_{k}
$$

where $a_{k}$ are complex numbers. Suppose $v$ satisfies the ODE

$$
v^{\prime}(t)=\lambda v(t)+\frac{\lambda a_{N}}{N}(1-t) T_{N}^{\prime}(t) .
$$

Note that the left-hand side of the above equation is a polynomial of degree $N-1$, while the first term at the right-hand side is a polynomial of degree $N$. The second term at the right-hand 
side is a polynomial of degree $N$ and has a constant factor chosen so that the right-hand side is a polynomial of degree $N-1$. Observe that

$$
v^{\prime}\left(t_{j}\right)=\lambda v\left(t_{j}\right), \quad 0 \leq j<N .
$$

When $0<j<N$, this is true because $T_{N}^{\prime}\left(t_{j}\right)=0$ by definition of the Chebyshev-Lobatto points. When $j=0$, then $t_{0}=1$, and the equality is obvious. (A.4) is equivalent to the relation

$$
[D] v\left(t_{h}\right)=\lambda v\left(t_{h}\right) .
$$

Using (A.2), equate the coefficient of $t^{N-1}$ on both sides of (A.3) to obtain

$$
a_{N-1}=2 a_{N}\left(\frac{N}{\lambda}-1\right) \text {. }
$$

Note that $\lambda \neq 0$ since otherwise, from (A.3) and the initial condition $v(-1)=0$, it would follow that $v \equiv 0$ and so $v\left(t_{h}\right)$ is the zero vector which cannot be an eigenvector. If $a_{N}=0$, then the left-hand side of (A.3) is a polynomial of degree one less than that at the right-hand side, which is impossible. Henceforth assume $a_{N} \neq 0$.

Let $\beta \in(0,1)$, whose value will be determined later. Multiply (A.4) by the factor $\rho_{j}\left(1-t_{j}\right)\left(1+\beta t_{j}\right) \overline{v\left(t_{j}\right)}$, then add the result to the complex conjugate of (A.4) multiplied by $\rho_{j}\left(1-t_{j}\right)\left(1+\beta t_{j}\right) v\left(t_{j}\right)$, and then sum the equation to obtain

$$
\sum_{j=0}^{N} \rho_{j} f\left(t_{j}\right)=2 R \sum_{j=0}^{N} \rho_{j}\left(1-t_{j}\right)\left(1+\beta t_{j}\right)\left|v\left(t_{j}\right)\right|^{2}:=C_{1} R,
$$

where $f(t)=(1-t)(1+\beta t)\left(|v|^{2}\right)^{\prime}(t)$ is a polynomial of degree $2 N+1$ and $\lambda=R+i S$ for real $R, S$. Note that we can extend the above sums to $j=N$ because both terms corresponding to $j=N$ vanish. Note also that $C_{1}$ is positive since otherwise $v\left(t_{j}\right)=0$, for $1 \leq j \leq N$. From (A.3), $v^{\prime}\left(t_{j}\right)=0$, for $1 \leq j \leq N$, and these conditions imply that $v \equiv 0$. In particular $v\left(t_{h}\right)=0$, and so it cannot be an eigenvector.

The goal is to show that the left-hand side of (A.6) is positive. To that end, write the left-hand side as $F+E$, where

$$
F=\int_{-1}^{1} f(t) w(t) d t, \quad E=\sum_{j=0}^{N} \rho_{j} f\left(t_{j}\right)-F .
$$

After integration by parts and some algebra,

$$
F=\int_{-1}^{1} \frac{1-\beta+\beta t+\beta t^{2}}{1+t}|v(t)|^{2} w(t) d t .
$$

It is easy to see that $1-\beta+\beta t+\beta t^{2} \geq c$, which is a positive constant for $\beta \in(0,4 / 5)$. Let $z(t)=v(t) / \sqrt{1+t}$, which is continuous on $[-1,1]$ since $v(-1)=0$. Thus

$$
F \geq c \int_{-1}^{1}|z(t)|^{2} w(t) d t=: C_{2} .
$$

Write

$$
f(t)=\sum_{k=0}^{2 N+1} b_{k} T_{k}(t)
$$


for some coefficients $b_{k}$. Then from Lemma A.1, $E=\pi b_{2 N}$. Thus $F+E \geq C_{2}+\pi b_{2 N}$.

Since the leading coefficient of $T_{k}$ is $2^{k-1}$ and the coefficient of $t^{k-1}$ of $T_{k}$ is zero, it follows that the coefficient of $t^{2 N}$ of $f$ is $2^{2 N-1} b_{2 N}$, which is equal to the coefficient of $t^{2 N}$ of the polynomial

$$
-2 \Re\left(a_{N} \overline{a_{N-1}}\right)\left(T_{N-1} T_{N}\right)^{\prime} \beta t^{2}-2(1-\beta)\left|a_{N}\right|^{2} T_{N} T_{N}^{\prime} t .
$$

From (A.2),

$$
b_{2 N} 2^{2 N-1}=-2^{2 N-2} \Re\left(a_{N} \overline{a_{N-1}}\right)(2 N-1) \beta-2^{2 N-1}\left|a_{N}\right|^{2}(1-\beta) N,
$$

or

$$
b_{2 N}=-\beta \Re\left(a_{N} \overline{a_{N-1}}\right) \frac{2 N-1}{2}-\left|a_{N}\right|^{2}(1-\beta) N .
$$

Substitute (A.5) to get

$$
b_{2 N}=\left|a_{N}\right|^{2}\left(-\beta N(2 N-1) \frac{R}{|\lambda|^{2}}+\beta(2 N-1)-(1-\beta) N\right) .
$$

Now (A.6) becomes

$$
C_{1} R=E+F \geq C_{2}+\pi b_{2 N}
$$

Substitute (A.7) into the above inequality to obtain

$$
\left(\frac{C_{1}}{\pi}+\frac{\left|a_{N}\right|^{2} \beta N(2 N-1)}{|\lambda|^{2}}\right) R \geq \frac{C_{2}}{\pi}+\left|a_{N}\right|^{2}(\beta(3 N-1)-N) .
$$

For any value of $\beta$ satisfying

$$
\frac{N}{3 N-1}<\beta<\frac{4}{5}
$$

it is possible to deduce that

$$
\left(\frac{C_{1}}{\pi}+\frac{2\left|a_{N}\right|^{2} N^{2}}{|\lambda|^{2}}\right) R \geq C_{3},
$$

for some positive constant $C_{3}$ independent of $N$. It can be concluded that $R>0$.

To show that $R$ is bounded away from zero, first note that from (A.2), for $0 \leq k \leq N$,

$$
a_{k}\left\|T_{k}\right\|_{0, w}^{2}=\int_{-1}^{1} v T_{k} w, \quad\|u\|_{0, w}^{2}=\int_{-1}^{1} u^{2} w,
$$

leading to

$$
\left|a_{k}\right| \leq \frac{\|v\|_{0, w}\left\|T_{k}\right\|_{0, w}}{\left\|T_{k}\right\|_{0, w}^{2}}<1,
$$

if we assume the normalization $\|v\|_{0, w}=1$. From (A.5), it follows that

$$
\left|a_{N-1}\right|^{2}=4\left|\frac{N}{\lambda}-1\right|^{2}\left|a_{N}\right|^{2}
$$


or

$$
\frac{\left|a_{N}\right|^{2} N^{2}}{|\lambda|^{2}}=\frac{\left|a_{N-1}\right|^{2} N^{2}}{4|N-\lambda|^{2}}<\frac{1}{4\left|1-\frac{\lambda}{N}\right|^{2}}=\frac{1}{4\left[\left(1-\frac{R}{N}\right)^{2}+\frac{S^{2}}{N^{2}}\right]}
$$

If $N=1$, then $[D]=1 / 2=R$. Henceforth, assume $N \geq 2$. If $R>1$, then we are done. Otherwise, assume $R \leq 1$. Then (A.9) becomes

$$
\frac{\left|a_{N}\right|^{2} N^{2}}{|\lambda|^{2}}<\frac{1}{4\left(1-\frac{1}{N}\right)^{2}} \leq 1
$$

since $N \geq 2$. Inserting this inequality into (A.8) immediately yields that $R \geq C_{4}$ with a positive constant independent of $N$.

Finally, we prove the remaining proposition.

Proof of Proposition 3.4. When $N=1,2$, the eigenvalues of $B^{T}$ are 1 and $(1 \pm i / \sqrt{3}) / 2$, respectively, and they have a positive real part. Henceforth, assume $N \geq 3$. Suppose $\left\{a_{k}\right\}$ is a set of complex constants so that

$$
v=\sum_{k=0}^{N} a_{k} T_{k}, \quad v(-1)=0
$$

with $\|v\|_{0, w}=1$, and where $v$ satisfies

$$
\int_{-1}^{t} v(\tau) d \tau=\lambda v(t)+\frac{a_{N}}{N(N+1)}\left(t^{2}-1\right) T_{N}^{\prime}(t)
$$

for $\lambda$ an eigenvalue of $B^{T}$. Note that the coefficient $a_{N} /(N(N+1))$ at the right-hand side of (A.11) has been chosen so that the coefficients of $T_{N+1}$ on both sides of (A.11) agree. It is easy to verify that $B^{T} v\left(t_{h}\right)=\lambda v\left(t_{h}\right)$. Using the identity

$$
\int_{-1}^{t} T_{k}(\tau) d \tau=\frac{1}{2}\left(\frac{T_{k+1}(t)}{k+1}-\frac{T_{k-1}(t)}{k-1}\right)+\frac{(-1)^{k+1}}{k^{2}-1}, \quad k \geq 2
$$

and on equating the coefficients of $T_{N}, T_{N-1}$, and $T_{N-2}$ on both sides of (A.11), we obtain (details will be given later)

$$
\begin{aligned}
\frac{a_{N-1}}{2 N} & =\lambda a_{N}, \\
\frac{a_{N-2}-a_{N}}{2(N-1)} & =\lambda a_{N-1}-\frac{a_{N}}{2(N+1)}, \\
\frac{a_{N-3}-a_{N-1}}{2(N-2)} & =\lambda a_{N-2} .
\end{aligned}
$$

(A.11) evaluated at $t=t_{j}$ reads

$$
\int_{-1}^{t_{j}} v(\tau) d \tau=\lambda v\left(t_{j}\right), \quad 0 \leq j \leq N
$$


Let $\beta \in(0,1)$, whose value will be determined later. Multiply (A.15) by the factor $\rho_{j}\left(1-t_{j}\right)\left(1-\beta t_{j}\right) \overline{v\left(t_{j}\right)}$, then add the result to the complex conjugate of (A.15) multiplied by $\rho_{j}\left(1-t_{j}\right)\left(1-\beta t_{j}\right) v\left(t_{j}\right)$, and then sum the equations to obtain

$$
\sum_{j=0}^{N} \rho_{j} f\left(t_{j}\right)=2 R \sum_{j=0}^{N} \rho_{j}\left(1-t_{j}\right)\left(1-\beta t_{j}\right)\left|v\left(t_{j}\right)\right|^{2}:=C_{2} R,
$$

where

$$
f(t)=(1-t)(1-\beta t)\left(\left|\int_{-1}^{t} v(\tau) d \tau\right|^{2}\right)^{\prime}
$$

is a polynomial of degree $2 N+3, R=\Re \lambda$, and $C_{2}$ is positive. Note that each term corresponding to $j=N$ in (A.16) vanishes since $v(-1)=0$. Write

$$
f(t)=\sum_{k=0}^{2 N+3} b_{k} T_{k}(t)
$$

for some complex $b_{k}$. By applying Lemma A.1, we have

$$
\int_{-1}^{1} f(t) w(t) d t+\pi b_{2 N}=C_{2} R
$$

After integration by parts, the above identity becomes

$$
\int_{-1}^{1} \frac{1+\beta-\beta t-\beta t^{2}}{1+t}\left|\int_{-1}^{t} v(\tau) d \tau\right|^{2} w(t) d t+\pi b_{2 N}=C_{2} R
$$

Use (A.10), (A.12), (A.13), (A.14) to obtain (details will be given later)

$$
\begin{aligned}
b_{2 N}=\frac{1}{2} \Re\left(\overline{a_{N}} a_{N-1}\right)\left(\frac{1}{N}+\frac{1}{N+1}\right) & (1+\beta)\left[\Re\left(\overline{a_{N}} a_{N-2}\right)\left(\frac{1}{N-1}+\frac{1}{N+1}\right)\right. \\
- & \left.\quad+\frac{1}{N}\left|a_{N-1}\right|^{2}-\frac{1}{N-1}\left|a_{N}\right|^{2}\right] \\
+ & \frac{\beta}{8}\left[\Re\left(\overline{a_{N}} a_{N-3}\right)\left(\frac{1}{N-2}+\frac{1}{N+1}\right)\right. \\
& +\Re\left(\overline{a_{N-1}} a_{N-2}\right)\left(\frac{1}{N-1}+\frac{1}{N}\right) \\
& \left.-\Re\left(\overline{a_{N}} a_{N-1}\right)\left(\frac{1}{N-2}+\frac{1}{N-1}\right)\right] \\
- & (1+\beta)\left|a_{N}\right|^{2}\left(\frac{c_{N+1}}{(N+1) 2^{N}}+\frac{c_{N}}{(N+1) 2^{N-1}}\right) \\
+ & \beta \Re\left(\overline{a_{N}} a_{N-1}\right)\left(\frac{c_{N+1}}{(N+1) 2^{N+1}}+\frac{c_{N}}{N 2^{N-1}}+\frac{c_{N-1}}{(N+1) 2^{N-1}}\right) .
\end{aligned}
$$

Here $c_{k}=-2^{k-3} k$ is the second leading coefficient of $T_{k}$ :

$$
T_{k}(t)=2^{k-1} t^{k}+c_{k} t^{k-2}+\cdots, \quad k \geq 2 .
$$


Substitute the expression for $b_{2 N}$ into (A.17) to obtain

$$
\int_{-1}^{1} \frac{1+\beta-\beta t-\beta t^{2}}{1+t}\left|\int_{-1}^{t} v(\tau) d \tau\right|^{2} w(t) d t+S_{1}=\left(S_{2}+C_{2}\right) R
$$

where

$$
\begin{aligned}
S_{1}= & \frac{(1+\beta)}{4} \frac{N-1}{(N+1)^{2}}\left|a_{N}\right|^{2}+\pi \frac{(1+\beta)}{4} \frac{N-1}{N(N+1)}\left|a_{N-1}\right|^{2} \\
& -\pi(1+\beta)\left(\frac{c_{N+1}}{(N+1) 2^{N}}+\frac{c_{N}}{(N+1) 2^{N-1}}\right)\left|a_{N}\right|^{2}>0, \\
S_{2}=\pi & S_{3}\left|a_{N}\right|^{2}+\frac{\pi \beta}{2} \frac{(2 N-1)(N-2)}{N(N+1)}\left|a_{N-1}\right|^{2} \\
& -2 \pi \beta N\left|a_{N}\right|^{2}\left[\frac{c_{N+1}}{(N+1) 2^{N+1}}+\frac{c_{N}}{N 2^{N-1}}+\frac{c_{N-1}}{(N+1) 2^{N-1}}\right],
\end{aligned}
$$

and

$$
S_{3}=-4 \beta \frac{N(2 N-1)(N-1)}{N+1} R^{2}+4(1+\beta) \frac{N^{2}}{N+1} R-\frac{3 \beta}{2} \frac{1}{(N+1)^{2}}-\frac{2 N+1}{N+1} .
$$

Note that the last term of the expression for $S_{2}$ is positive since $c_{k}<0$ and the coefficient of $\left|a_{N-1}\right|^{2}$ is positive. Note also that the integral at the left-hand side of (A.20) is positive for $\beta \in(0,1)$. Hence the remaining goal is to choose $\beta$ so that $S_{3}$, a quadratic in $R$, is positive. Toward that end, the maximum of $S_{3}$ occurs at

$$
R=\frac{1+\beta}{2 \beta} \frac{N}{(2 N-1)(N-1)}
$$

with maximum value

$$
\frac{(1+\beta)^{2}}{\beta} \frac{N^{3}}{(2 N-1)\left(N^{2}-1\right)}-\frac{3 \beta}{2} \frac{N}{(N+1)^{2}}-\frac{2 N+1}{N+1} .
$$

Hence we require

$$
\frac{(1+\beta)^{2}}{\beta} \frac{N^{3}}{(2 N-1)(N-1)}-\frac{3 \beta}{2} \frac{N}{N+1}>2 N+1 .
$$

Notice that for $N \geq 3$,

$$
\frac{N^{2}}{(2 N-1)(N-1)}>\frac{1}{2}, \quad \frac{3}{N+1}<1 .
$$

Assume that $\beta<1 / 3$, then $1+\beta>4 \beta$ and

$$
\frac{(1+\beta)^{2}}{\beta} \frac{N^{3}}{(2 N-1)(N-1)}-\frac{\beta}{2} \frac{3 N}{N+1}>\frac{15}{2} \beta N .
$$

Therefore it is enough to choose $\beta$ so that

$$
\frac{4 N+2}{15 N}<\beta<\frac{1}{3}
$$


With this choice of $\beta$, it follows from (A.20) that $R>0$.

Next we supply some details of the above calculations. First we prove (A.12)-(A.14). Apply (A.10) in (A.11) to obtain

$$
\sum_{k=0}^{N} a_{k} \int_{-1}^{t} T_{k}(\tau) d \tau=\lambda \sum_{k=0}^{N} a_{k} T_{k}(t)+\frac{a_{N}}{N(N+1)}\left(t^{2}-1\right) T_{N}^{\prime}(t)
$$

The left-hand side of this equation is

$$
\begin{aligned}
& \sum_{k=0}^{N} a_{k} \int_{-1}^{t} T_{k}(\tau) d \tau=a_{N} \int_{-1}^{t} T_{N}(\tau) d \tau+a_{N-1} \int_{-1}^{t} T_{N-1}(\tau) d \tau \\
& \quad+a_{N-2} \int_{-1}^{t} T_{N-2}(\tau) d \tau+a_{N-3} \int_{-1}^{t} T_{N-3}(\tau) d \tau+\cdots \\
& =\frac{1}{2} a_{N}\left[\frac{T_{N+1}}{N+1}-\frac{\left.T_{N-1}\right]}{N-1]}+\frac{1}{2} a_{N-1}\left[\frac{T_{N}}{N}-\frac{T_{N-2}}{N-2}\right]\right. \\
& \quad+\frac{1}{2} a_{N-2}\left[\frac{T_{N-1}}{N-1}-\frac{T_{N-3}}{N-3}\right]+\frac{1}{2} a_{N-3}\left[\frac{T_{N-2}}{N-2}-\frac{T_{N-4}}{N-4}\right]+\cdots \\
& =\frac{a_{N}}{2(N+1)} T_{N+1}+\frac{a_{N-1}}{2 N} T_{N}+\left(\frac{a_{N-2}}{2(N-1)}-\frac{a_{N}}{2(N-1)}\right) T_{N-1} \\
& +\left(\frac{a_{N-3}}{2(N-2)}-\frac{a_{N-1}}{2(N-2)}\right) T_{N-2}+\cdots \cdot
\end{aligned}
$$

The second term at the right-hand side of (A.21) is

$$
\begin{aligned}
& \frac{a_{N}}{N(N+1)}\left(t^{2}-1\right) T_{N}^{\prime}(t)= \frac{a_{N}}{(N+1)}\left(t^{2}-1\right)\left[\frac{T_{N}^{\prime}(t)}{N}-\frac{T_{N-2}^{\prime}(t)}{N-2}\right. \\
&\left.\quad+\frac{T_{N-2}^{\prime}(t)}{N-2}-\frac{T_{N-4}^{\prime}(t)}{N-4}+\frac{T_{N-4}^{\prime}(t)}{N-4}\right] \\
&= \frac{a_{N}}{(N+1)} 2\left(t^{2}-1\right)\left[T_{N-1}+T_{N-3}+\frac{T_{N-4}^{\prime}(t)}{2(N-4)}\right] \\
&= \frac{a_{N}}{(N+1)}\left[2 t^{2} T_{N-1}+2 t^{2} T_{N-3}-2 T_{N-1}-2 T_{N-3}+\cdots\right] \\
&= \frac{a_{N}}{(N+1)}\left[t\left(T_{N}+T_{N-2}\right)+t\left(T_{N-2}+T_{N-4}\right)\right. \\
&= \frac{a_{N}}{(N+1)}\left[\frac { 1 } { 2 } \left(T_{N+1}+T_{N-1}+T_{N-1}+T_{N-3}\right.\right. \\
&\left.+T_{N-1}+T_{N-3}+T_{N-3}+T_{N-5}\right) \\
&\left.-2 T_{N-1}-2 T_{N-3}+\cdots\right] .
\end{aligned}
$$


So the right-hand side of (A.21) becomes

$$
\begin{gathered}
\lambda \sum_{k=0}^{N} a_{k} T_{k}(t)+\frac{a_{N}}{N(N+1)}\left(t^{2}-1\right) T_{N}^{\prime}(t) \\
=\lambda a_{N} T_{N}(t)+\lambda a_{N-1} T_{N-1}(t)+\lambda a_{N-2} T_{N-2}(t)+\ldots \\
\quad+\frac{a_{N}}{(N+1)}\left[\frac{1}{2}\left(T_{N+1}+3 T_{N-1}+4 T_{N-3}+\cdots\right)\right. \\
\left.-2 T_{N-1}-2 T_{N-3}+\cdots\right] \\
=\frac{a_{N}}{2(N+1)} T_{N+1}+\lambda a_{N} T_{N}(t)+\left[\lambda a_{N-1}-\frac{a_{N}}{2(N+1)}\right] T_{N-1}(t) \\
\quad+\lambda a_{N-2} T_{N-2}(t)+\cdots .
\end{gathered}
$$

Therefore by equating the coefficients of equations (A.22) and (A.23), we arrive at (A.12)(A.14).

Next we show that the following equalities follow from (A.12)-(A.14):

$$
\begin{aligned}
\frac{1}{2 N} \Re\left(a_{N-1} \overline{a_{N}}\right) & =R\left|a_{N}\right|^{2} \\
\frac{1}{2(N-1)} \Re\left(a_{N-2} \overline{a_{N-1}}\right) & =R\left(\left|a_{N-1}\right|^{2}+\frac{2 N}{N^{2}-1}\left|a_{N}\right|^{2}\right) \\
\frac{1}{N-1} \Re\left(a_{N-2} \overline{a_{N}}\right) & =\left(8 N R^{2}+\frac{2}{N^{2}-1}\right)\left|a_{N}\right|^{2}-\frac{1}{N}\left|a_{N-1}\right|^{2} \\
\frac{1}{N-2} \Re\left(a_{N-3} \overline{a_{N}}\right) & =\left(32 N(N-1) R^{3}+\left[\frac{4}{N+1}+\frac{2 N}{N-2}\right] R\right)\left|a_{N}\right|^{2} \\
& -\frac{6(N-1)}{N} R\left|a_{N-1}\right|^{2}
\end{aligned}
$$

Observe that (A.24) follows directly from (A.12). From (A.13), we find

$$
\frac{1}{2(N-1)} a_{N-2}=\lambda a_{N-1}+\frac{1}{N^{2}-1} a_{N} .
$$

Use this equation and (A.12) to arrive at (A.25) and

$$
\frac{1}{N-1} \Re\left(a_{N-2} \overline{a_{N}}\right)=\lambda a_{N-1} \overline{a_{N}}+\bar{\lambda} \overline{a_{N-1}} a_{N}+\frac{2}{N^{2}-1}\left|a_{N}\right|^{2} .
$$

Also from (A.12)

$$
\frac{1}{N}\left|a_{N-1}\right|^{2}=\lambda a_{N} \overline{a_{N-1}}+\bar{\lambda} \overline{a_{N}} a_{N-1} .
$$

Add (A.28) and (A.29), and use (A.24) to recover (A.26). The following equations follow from (A.14) and (A.12), respectively:

$$
\frac{1}{N-2} \Re\left(a_{N-3} \overline{a_{N}}\right)=\lambda a_{N-2} \overline{a_{N}}+\bar{\lambda} \overline{a_{N-2}} a_{N}+\frac{1}{N-2} \Re\left(a_{N} \overline{a_{N-1}}\right),
$$

and

$$
\frac{1}{N} \Re\left(a_{N-1} \overline{a_{N-2}}\right)=\lambda a_{N} \overline{a_{N-2}}+\bar{\lambda} \overline{a_{N}} a_{N-2} .
$$


Add (A.30) and (A.31) to get

$$
\frac{1}{N-2} \Re\left(a_{N-3} \overline{a_{N}}\right)+\frac{1}{N} \Re\left(a_{N-1} \overline{a_{N-2}}\right)=4 R \Re\left(a_{N} \overline{a_{N-2}}\right)+\frac{1}{N-2} \Re\left(a_{N} \overline{a_{N-1}}\right) .
$$

Combine the above, (A.24), (A.25), and (A.26) to get (A.27).

Finally we derive the expression for $b_{2 N}$. Apply (A.10) and performing some calculations leads to

$$
\begin{aligned}
& \bar{v} \int v+v \int \bar{v} \\
& =2\left|a_{N}\right|^{2}\left(T_{N} \int T_{N}\right)+2\left|a_{N-1}\right|^{2}\left(T_{N-1} \int T_{N-1}\right) \\
& +2 \Re\left(\overline{a_{N}} a_{N-1}\right)\left(T_{N} \int T_{N-1}+T_{N-1} \int T_{N}\right) \\
& +2 \Re\left(\overline{a_{N}} a_{N-2}\right)\left(T_{N} \int T_{N-2}+T_{N-2} \int T_{N}\right) \\
& +2 \Re\left(\overline{a_{N}} a_{N-3}\right)\left(T_{N} \int T_{N-3}+T_{N-3} \int T_{N}\right) \\
& +2 \Re\left(\overline{a_{N-1}} a_{N-2}\right)\left(T_{N-1} \int T_{N-2}+T_{N-2} \int T_{N-1}\right)+\cdots \\
& =\left|a_{N}\right|^{2}\left(T_{N}\left[\frac{T_{N+1}}{N+1}-\frac{T_{N-1}}{N-1}\right]\right)+\left|a_{N-1}\right|^{2}\left(T_{N-1}\left[\frac{T_{N}}{N}-\frac{T_{N-2}}{N-2}\right]\right) \\
& +\Re\left(\overline{a_{N}} a_{N-1}\right)\left(T_{N}\left[\frac{T_{N}}{N}-\frac{T_{N-2}}{N-2}\right]+T_{N-1}\left[\frac{T_{N+1}}{N+1}-\frac{T_{N-1}}{N-1}\right]\right) \\
& +\Re\left(\overline{a_{N}} a_{N-2}\right)\left(T_{N}\left[\frac{T_{N-1}}{N-1}-\frac{T_{N-3}}{N-3}\right]+T_{N-2}\left[\frac{T_{N+1}}{N+1}-\frac{T_{N-1}}{N-1}\right]\right) \\
& +\Re\left(\overline{a_{N}} a_{N-3}\right)\left(T_{N}\left[\frac{T_{N-2}}{N-2}-\frac{T_{N-4}}{N-4}\right]+T_{N-3}\left[\frac{T_{N+1}}{N+1}-\frac{T_{N-1}}{N-1}\right]\right) \\
& +\Re\left(\overline{a_{N-1}} a_{N-2}\right)\left(T_{N-1}\left[\frac{T_{N-1}}{N-1}-\frac{T_{N-3}}{N-3}\right]+T_{N-2}\left[\frac{T_{N}}{N}-\frac{T_{N-2}}{N-2}\right]\right)+\cdots,
\end{aligned}
$$

where $\cdots$ denotes the remaining parts in the expansion. By (A.19),

$$
\begin{gathered}
\bar{v}(t) \int_{-1}^{t} v(\tau) d \tau+v(t) \int_{-1}^{t} \bar{v}(\tau) d \tau \\
=\Re\left(\overline{a_{N}} a_{N-1}\right)\left(\frac{1}{N}+\frac{1}{N+1}\right) 2^{2 N-2} t^{2 N} \\
+\left[\Re\left(\overline{a_{N}} a_{N-2}\right)\left(\frac{1}{N-1}+\frac{1}{N+1}\right)\right. \\
\left.\quad+\frac{1}{N}\left|a_{N-1}\right|^{2}-\frac{1}{N-1}\left|a_{N}\right|^{2}\right] 2^{2 N-3} t^{2 N-1} \\
+\quad\left[\Re\left(\overline{a_{N}} a_{N-3}\right)\left(\frac{1}{N-2}+\frac{1}{N+1}\right)+\Re\left(\overline{a_{N-1}} a_{N-2}\right)\left(\frac{1}{N-1}+\frac{1}{N}\right)\right. \\
\left.\quad-\Re\left(\overline{a_{N}} a_{N-1}\right)\left(\frac{1}{N-2}+\frac{1}{N-1}\right)\right] 2^{2 N-4} t^{2 N-2}
\end{gathered}
$$




$$
\begin{aligned}
& +\left|a_{N}\right|^{2}\left(\frac{c_{N+1}}{N+1} 2^{N-1}+\frac{c_{N}}{N+1} 2^{N}\right) t^{2 N-1} \\
& +\Re\left(\overline{a_{N}} a_{N-1}\right)\left(\frac{c_{N+1}}{N+1} 2^{N-2}+\frac{c_{N}}{N} 2^{N}+\frac{c_{N-1}}{N+1} 2^{N}\right) t^{2 N-2}+\cdots
\end{aligned}
$$

Then

$$
\begin{aligned}
& f(t)=\left(1-(1+\beta) t+\beta t^{2}\right)\left(\bar{v}(t) \int_{-1}^{t} v(\tau) d \tau+v(t) \int_{-1}^{t} \bar{v}(\tau) d \tau\right) \\
& =\Re\left(\overline{a_{N}} a_{N-1}\right)\left(\frac{1}{N}+\frac{1}{N+1}\right) 2^{2 N-2} t^{2 N} \\
& -(1+\beta)\left[\Re\left(\overline{a_{N}} a_{N-2}\right)\left(\frac{1}{N-1}+\frac{1}{N+1}\right)\right. \\
& \left.+\frac{1}{N}\left|a_{N-1}\right|^{2}-\frac{1}{N-1}\left|a_{N}\right|^{2}\right] 2^{2 N-3} t^{2 N} \\
& +\beta\left[\Re\left(\overline{a_{N}} a_{N-3}\right)\left(\frac{1}{N-2}+\frac{1}{N+1}\right)+\Re\left(\overline{a_{N-1}} a_{N-2}\right)\left(\frac{1}{N-1}+\frac{1}{N}\right)\right. \\
& \left.-\Re\left(\overline{a_{N}} a_{N-1}\right)\left(\frac{1}{N-2}+\frac{1}{N-1}\right)\right] 2^{2 N-4} t^{2 N} \\
& \quad-(1+\beta)\left|a_{N}\right|^{2}\left(\frac{c_{N+1}}{N+1} 2^{N-1}+\frac{c_{N}}{N+1} 2^{N}\right) t^{2 N} \\
& +\beta \Re\left(\overline{a_{N}} a_{N-1}\right)\left(\frac{c_{N+1}}{N+1} 2^{N-2}+\frac{c_{N}}{N} 2^{N}+\frac{c_{N-1}}{N+1} 2^{N}\right) t^{2 N} \\
& =2^{2 N-1} b_{2 N} t^{2 N}+\cdots
\end{aligned}
$$

where $b_{2 N}$ is given by (A.18).

\section{REFERENCES}

[1] R. H. Bartels And G. W. Stewart, Algorithm 432: Solution of the matrix equation $A X+X B=C$, Comm. ACM, 15 (1972), pp. 820-826.

[2] C. Canuto, M. Y. Hussaini, A. Quarteroni, And T. A. Zang, Spectral Methods: Fundamentals in Single Domains, Springer, Berlin, 2006.

[3] M. J. GANDER, 50 years of time parallel time integration, in Multiple Shooting and Time Domain Decomposition Methods, T. Carraro, M. Geiger, S. Körkel, and R. Rannachev, eds., Contrib. Math. Comput. Sci.,9, Springer, Cham, 2015, pp. 69-113.

[4] B.-Y. GUO AND Z.-Q. WANG, Legendre-Gauss collocation methods for ordinary differential equations, Adv. Comput. Math., 30 (2009), pp. 249-280.

[5] J.-G. TANG AND H.-P. MA, A Legendre spectral method in time for first-order hyperbolic equations, Appl. Numer. Math., 57 (2007), pp. 1-11.

[6] G. IERley, B. SPEnCER, AND R. Worthing, Spectral methods in time for a class of parabolic partial differential equations, J. Comput. Phys., 102 (1992), pp. 88-97.

[7] W. LiU, B. Wu, AND J. Sun, Space-time spectral collocation method for the one-dimensional Sine-Gordon equation, Numer. Methods Partial Differential Equations, 31 (2015), pp. 670-690.

[8] S. H. LuI, Numerical Analysis of Partial Differential Equations, Wiley, Hoboken, 2011.

[9] _ Legendre spectral collocation in space and time for PDEs, Numer. Math., 136 (2017), pp. 75-99.

[10] S. H. LUI AND S. NATAJ, Spectral collocation in space and time for linear PDEs, Preprint, submitted, 2020

[11] S. NATAJ, Topics in Quasi-Newton and Space-time Spectral Methods, PhD. Thesis, Faculty of Graduate Studies, University of Manitoba, Winnipeg, 2019.

[12] S. C. REDDY AND J. A. C. WEIDEMAN, The accuracy of the Chebyshev differencing method for analytic functions, SIAM J. Numer. Anal., 42 (2005), pp. 2176-2187.

[13] J. SHEN AND L.-L. WANG, Fourierization of the Legendre-Galerkin method and a new space-time spectral method, Appl. Numer. Math., 57 (2007), pp. 710-720. 
ETNA

Kent State University and

Johann Radon Institute (RICAM)

[14] A. Solomonoff And E. Turkel, Global properties of pseudospectral methods, J. Comput. Phys., 81 (1989), pp. 239-276.

[15] A. Y. SuHOV, A spectral method for the time evolution in parabolic problems, J. Sci. Comput., 29 (2006), pp. 201-217.

[16] H. TAL-EZER, Spectral methods in time for hyperbolic equations, SIAM J. Numer. Anal., 23 (1986), pp. 11-26.

[17] - Spectral methods in time for parabolic problems, SIAM J. Numer. Anal., 26 (1989), pp. 1-11.

[18] J. G. TANG AND H. P. MA, Single and multi-interval Legendre $\tau$-methods in time for parabolic equations, Adv. Comput. Math., 17 (2002), pp. 349-367.

[19] T. TANG AND X. XU, Accuracy enhancement using spectral postprocessing for differential equations and integral equations, Commun. Comput. Phys., 5 (2009), pp. 779-792.

[20] A. TOWNSEND AND S. OLVER, The automatic solution of partial differential equations using a global spectral method, J. Comput. Phys., 299 (2015), pp. 106-123.

[21] L. N. TREFETHEN AND J. A. C. WeIDEMAn, The eigenvalues of second-order spectral differentiation matrices, SIAM J. Numer. Anal., 25 (1988), pp. 1279-1298.

[22] L. YI AND Z. WANG, Legendre-Gauss type spectral collocation algorithms for nonlinear ordinary partial differential equations, Int. J. Comput. Math., 91 (2014), pp. 1434-1460.

[23] - Legendre spectral collocation method for second-order nonlinear ordinary partial differential equations, Discrete Contin. Dyn. Syst. Ser. B, 19 (2014), pp. 299-322. 